\title{
La alimentación responsiva en la aceptación de nuevos alimentos en niños de 2 a 3 años
}

\author{
Responsive feeding in the accepting of new food in children from 2 to 3 years old
}

DOI: 10.33539/consensus.2019.v24n2.2323

Romina Jarama Redshaw ${ }^{1}$

Marinalva Santos Bandy²

\begin{abstract}
RESUMEN
El estudio busca demostrar que el empleo de la Técnicas de Alimentación Responsiva contribuye a la mejora de hábitos alimentarios a través de la aceptación de nuevos alimentos en niños de 2 a 3 años de edad. El estudio es de tipo aplicado, de nivel descriptivo y de diseño transeccional descriptivo. La población estuvo conformada por 66 preescolares de la I.E.I. "Niño Jesús de Praga", 2014. Se aplicó el instrumento de observación para cuidadoras, The observation instrument for caregivers. Se empleó la prueba estadística Chi Cuadrado para medir el nivel de significancia del estudio. Los resultados indican que la aplicación de las técnicas facilita el consumo de nuevos alimentos en la dieta de los preescolares, y por lo tanto, se puede afirmar que la aceptación de los nuevos alimentos con el empleo de las Técnicas de Alimentación Responsiva a los niños entre de 2 a 3 años de edad, es efectiva $(p<0.0001)$.
\end{abstract}

Palabras clave

Alimentación Responsiva, ingesta de nuevos alimentos, preescolares, cuidadoras, hábitos alimentarios saludables

\section{ABSTRACT}

The study seeks to prove that the use of Techniques of Responsive Feeding contributes to improvement of eating habits through the acceptance of new foods in children 2 to 3 years old. The study is an applied type, descriptive level and a cross-sectional descriptive design. The population was consisted of 66 preschool children from an educational institution, called I.E.I. "Niño Jesús de Praga", in 2014. The observation instrument for caregivers was applied. In addition, the Chi-square statistical test was employed to measure the significance level of the study. The results indicate that the application of techniques facilitates the consumption of new foods in the diet of preschool children, and therefore, it could be asserted that the accepting of new foods, and the use of the Techniques of Responsive Feeding to children from 2 to 3 years old, it is effective $(p<0.0001)$.

\section{Keywords}

Responsive feeding techniques, new food intake, preschoolers, caregivers, healthy eating habits

1 Licenciada en Nutrición y Dietética por la Universidad Femenina del Sagrado Corazón, UNIFÉ, Lima. Estudiante de maestría en Gestión y Docencia en Alimentación y Nutrición.

2 Doctora en Salud Publica; Magister Scientiae - MSc. en Nutrición; Licenciada en Nutrición y Dietética; Decana de la Facultad de Nutrición y Alimentación - Universidad Femenina del Sagrado Corazón- UNIFE. Creadora del Programa Nutrición Inteligente- NUTRAZONE. 


\section{INTRODUCCIÓN}

El diagnóstico del estado nutricional de la mayoría de niños menores de 5 años de edad en el Perú presentan actualmente un crecimiento en la tasa de sobrepeso y obesidad, observándose una disminución en la tasa de desnutrición (Instituto Nacional de estadística e Informática [INEI], 2010, 2013; Instituto Nacional de Salud [INS], 2015). Estos resultados podrían describir unas prácticas inadecuadas en la manera de alimentar a la población infantil, la baja oferta de alimentos variados a los niños y desconocimiento de la existencia de técnicas de alimentación que pueden mejorar la aceptación de nuevos alimentos que promueva una gran variedad de nutrientes, sabores, texturas diferentes y un ambiente agradable al niño.

Black y Creed (2012) y Macías, Gordillo y Camacho (2012) coinciden en que los hábitos en la alimentación se forman durante la infancia, de allí la importancia del empleo de técnicas de alimentación responsiva que promueva un momento de armonía y estímulo al niño para aceptar una dieta variada, con nuevos alimentos que permita recibir los nutrientes que su cuerpo necesita para un crecimiento y desarrollo normal.

El objetivo de la presente investigación fue demostrar que, la aplicación de las técnicas de alimentación responsiva contribuye a la mejora de hábitos alimentarios a través de la aceptación de nuevos alimentos en niños de 2 a 3 años de edad, por lo que, para los efectos de la presente investigación, se ofreció a este grupo de edad tres alimentos (kiwi, camu camu y aguaymanto) no consumidos por la mayor parte de los niños en esta etapa. Se presentó tres diferentes tipos de preparaciones por alimento durante 9 días útiles, con el fin de observar su aceptación a través de la aplicación de las técnicas de alimentación responsiva.

La alimentación responsiva es un término relativamente nuevo y es definida por diferentes autores y organizaciones que señalamos a continuación:

1. Según Black y Creed (2012) la alimentación responsiva brinda a los cuidadores la capacidad para leer y responder apropiadamente a las señales de hambre y saciedad de los niños.

2. Según Abaud y Black (2011) la alimentación responsiva incluye lo siguiente: 1) asegurar que el ambiente sea placentero y disminuir las distracciones; que el niño esté sentado cómodamente, idealmente mirando a otros; las expectativas son, la comunicación clara, y que la comida sea saludable, sabrosa, preparada apropiadamente, y que se ofrezca en un horario en el que el niño tenga hambre; 2) alentar y atender a las señales de hambre y saciedad; y 3) responder al niño con apremio, soporte emocional, y de una manera adecuada.

3. Con respecto a la definición que brinda la Organización Panamericana de la Salud (OPS, 2009), esta especifica cinco puntos claves sobre la alimentación perceptiva o responsiva:

a) alimentar a los lactantes directamente y asistir a los niños mayores cuando comen por sí solos, respondiendo a sus signos de hambre y satisfacción; b) alimentar despacio y pacientemente y animar 
a los niños a comer, pero sin forzarlos; c) si los niños rechazan varios alimentos, experimentar con diversas combinaciones, sabores, texturas y métodos para animarlos a comer; d) minimizar las distracciones durante las horas de comida si el niño pierde interés rápidamente; e) recordar que los momentos de comer son periodos de aprendizaje y amor -hablar con los niños y mantener el contacto visual. (p. 33)

4. Para la guía denominada Proceso para la Promoción de la Alimentación del Niño, ProPAN (OPS, 2013), la alimentación responsiva incluye cuatro dimensiones:

1) Animar adecuadamente al niño que no ha comido lo suficiente; 2) Hablar con el niño (sin ordenarle ni amenazarle durante las comidas; 3) Darle oportunidades al niño para que coma por sí mismo, aunque sea un poco, y 4) Ausencia de respuestas inapropiadas a un niño (por lo general relacionadas con la negativa a comer, aunque no siempre). (p. 62)

Complementando las definiciones anteriores, los autores Engle, Bentle y Pelto (2000) y Pelto, Levitt y Thairu (2003) se refieren a la alimentación responsiva como la manera de cómo, dónde, cuándo y quién alimenta al niño, confirmando que un estilo activo de alimentación mejora el consumo variado de alimentos.

La introducción de nuevos alimentos a la dieta de los niños se debe ofrecer de manera cautelosa, ya que Black y Creed (2012), en el artículo ¿Cómo alimentar a los niños? La práctica de conductas alimentarias saludables desde la infancia, relacionan los estilos de alimentar a los niños con la ingesta de alimentos, obteniendo que, cuando las cuidadoras emplean un estilo exigente donde predomina el control, presiona al niño a través de una alimentación forzada se relaciona con la baja ingesta de alimentos; por otro lado, un estilo de alimentación restrictiva donde la cuidadora priva de alimentos al niño, genera mayor ansiedad y el niño tiende a una ingesta excesiva de alimentos, y; por último, la alimentación indulgente donde la cuidadora es indiferente ante la alimentación del niño se relaciona con un aumento en la ingesta y ganancia de peso. Es así como la alimentación responsiva resulta ser la mejor alternativa en cuanto al consumo de alimentos y la mejora de hábitos alimentarios, es decir que es el estilo en donde la cuidadora responde a señales de hambre y saciedad del niño, logrando que consuma los alimentos necesarios en un ambiente estructurado y un clima emocional positivo.

Sin dejar de mencionar que, para ofrecer un nuevo alimento, se debe observar aisladamente las reacciones alérgicas que puede presentar al niño cada nuevo alimento.

\section{MÉTODO}

El presente estudio corresponde a una investigación de tipo aplicada, nivel descriptivo, diseño no experimental: transeccional descriptivo.

Se estudió a 66 preescolares, población total, cuyas edades se encuentran entre los 2 y 3 años, matriculados en las aulas de 2 
años de edad de la I.E.I. "Niño Jesús de Praga", ubicada en el distrito de San Borja.

Se aplicó el Instrumento de observación de alimentación perceptiva para cuidadoras que se encuentra en ProPAN (OPS, 2013), utilizado para la recopilación de datos, el cual consta de 13 preguntas cerradas referidas a las acciones realizadas por las cuidadoras durante el tiempo de la alimentación, siete de las cuales tienen puntaje según se realice o no la acción indicada. El puntaje sirve para evaluar si la cuidadora aplica las técnicas de alimentación responsiva. Se dice que se aplican las técnicas de alimentación responsiva cuando el puntaje final resulta mayor a 2.

La población de estudio fue evaluada durante el refrigerio, con el fin de observar la aplicación de las técnicas de alimentación responsiva y cómo estas lograban que el niño acepte los nuevos alimentos presentados. El registro se realizó a través de cámaras de video, debido a que se trataba de observar a más de un niño a la vez y tener un registro que permitiera una evaluación cualitativa de mayor precisión.

\section{Trabajo de campo}

1. La institución I.E.I. "Niño Jesús de Praga” autorizó la realización del trabajo de investigación, una vez obtenido el consentimiento informado se realizó el estudio.

2. Los alimentos seleccionados, aguaymanto, kiwi у сати сатu, fueron ofrecidos en tres diferentes preparaciones durante el refrigerio de los niños en estudio durante un periodo de 9 días útiles consecutivos. Al ser los tres alimentos frutas, se ofrecieron a los niños en el mismo horario establecido por la institución como horario de refrigerio, donde se ofrece a los niños jugo o fruta. Esto permitió que el estudio de observación se desarrollara de manera más efectiva, ya que las cuidadoras de la alimentación de los niños tenían conocimiento que la grabación era para observar el consumo de los nuevos alimentos, más no para observar sus acciones de cómo alimentar al niño con nuevos sabores y texturas de alimentos.

3. Con el apoyo de un grupo de seis (6) expertos, especializados en grabación con cámaras de video, y capacitados para grabar de forma discreta la manera en que se brindaba el alimento al niño y el comportamiento de este frente al nuevo alimento (frutas), se grabó a todas y cada una de las responsables de la alimentación del niño, especialmente en el momento que ofrecían las frutas preparadas de diferentes formas, y se pudo medir cuántos puntos de las técnicas de la alimentación responsiva empleó individualmente cada una de las cuidadoras mientras ofrecían los alimentos nuevos a los niños.

4. Simultáneamente, se entregó un formato de consumo de las preparaciones con los nuevos alimentos del estudio ofrecidos a los niños a las cuidadoras, para que ellas pudiesen señalar el consumo de estos alimentos, identificando el alimento y la cantidad consumida. Además, el formato incluía preguntas para obtener datos personales sobre las cuidadoras; incluía nueve hojas anexas con un formato listo para completar la preparación correspondiente a cada día, el nombre del aula y de los niños, y cuatro opciones de proporciones de la preparación consumida por el niño para que la cuidadora pueda marcarlos. 
5. Las preparaciones se entregaron en vasos plásticos descartables de $3 \mathrm{oz}(90 \mathrm{ml})$, los cuales llegaban al aula marcados con plumón indeleble en la mitad de estos para que la cuidadora pueda identificar rápidamente la proporción. En el caso de las frutas en trozos o enteras, se ofrecían cantidades impares con el mismo propósito de identificar la proporción.

\section{RESULTADOS}

La aplicación de las técnicas de alimentación responsiva por parte de las cuidadoras resultó clave para la aceptación de nuevos alimentos, ya que el $85 \%$ de la población estudiada logró introducirlos adecuadamente, mientras que, los niños alimentados por las cuidadoras que no aplicaron las técnicas de alimentación obtuvieron un rechazo del 55\%.

Las técnicas de alimentación responsiva se caracterizan por reconocer el hambre y saciedad de los niños por las cuidadoras (Black y Creed, 2012), es por ello que se consideró que el alimento era "aceptado" cuando el niño había consumido "más de la mitad de la porción", ya que muchas veces no consumía la totalidad del alimento o preparación porque ya estaba satisfecho y la cuidadora podía leerlo.

Las acciones realizadas por las cuidadoras se clasificaron según las dimensiones de la alimentación responsiva y la relación que tenían estas sobre el consumo del nuevo alimento, para poder conocer el efecto que tiene cada dimensión independientemente.

En la primera dimensión, "Animar adecuadamente al niño que no ha comido lo suficiente", las acciones observadas más utilizadas fueron las de hablarle al niño para llamar su atención a través de: "Le dice, le ruega, le pide que coma, le ordena suavemente", "Le dice que la comida es buena o le habla sobre la comida o pregunta sobre la comida" y "Le habla sobre otras cosas al niño, cuenta cuentos" en el orden mencionado. Sin embargo, las acciones que resultaron más efectivas en cuanto a la ingesta de nuevos alimentos y a la aplicación de las técnicas de alimentación responsiva fueron: "Le canta canciones o le dice que le va a cantar canciones", "Otra persona ayuda a comer al niño o le da cosas", "Le habla sobre otras cosas al niño, cuenta cuentos", "Juega con o sin juguetes, juega con o sin la cuidadora: juega, ríe con el niño para hacer que coma, da cosas o juguetes para que juegue, permite que coja cosas" y "Elogia al niño, acaricia, toca, besa para animarlo a que coma".

En la dimensión "Hablar con el niño (sin ordenarle ni amenazarle durante las comidas)", sucede algo similar que la dimensión anterior, solo que en esta dimensión, la función de hablar con el niño no será porque no quiere comer sino de proveer un ambiente armonioso. Las acciones más empleadas por las cuidadoras fueron "Le dice, le ruega, le pide que coma, le ordena suavemente", "Le dice que la comida es buena o le habla sobre la comida o pregunta sobre la comida" y "Le habla sobre otras cosas al niño, cuenta cuentos". En esta dimensión la acción más efectiva tanto para la aplicación de las técnicas de alimentación responsiva como para la aceptación de los alimentos fue "Le canta canciones o le dice que le va a cantar canciones" y "Elogia al niño, acaricia, toca, besa para animarlo a que coma".

En la dimensión "Darle oportunidades al niño para que coma por sí mismo, aunque 
sea un poco", la mayor parte de los niños (64\%) lo logró, puesto que el entorno físico de todos durante el refrigerio fue adecuado, ya que se les vio sentados en sillas pequeñas de acuerdo a su tamaño, logrando que los pies alcancen el suelo, y la mesa se hallara a la altura de su pecho.

La cuarta dimensión abarca la "ausencia de respuestas inapropiadas a un niño", se reconoció las respuestas inapropiadas para poder descontarlas al puntaje. Se encontró que la acción más repetida fue "No hizo nada, no ofreció más", es decir no insistió para que el niño consuma el alimento aun cuando no había comido lo suficiente. Las acciones más comunes fueron "la cuidadora tiene que sujetar al niño frecuentemente" e "Insiste, persigue, no da tiempo al niño, acosa".

En la población estudiada se encontró que, el $85 \%$ del grupo de niños que consumieron igual o más de mitad de los alimentos ofrecidos durante toda la investigación fueron alimentados aplicando las técnicas de alimentación responsiva. Dentro del grupo de niños en el que las cuidadoras no aplicaron las técnicas, se evidenció un 45\% de aceptación. Entonces, ello refleja que existe una diferencia del $40 \%$ de niños que, gracias a las técnicas de alimentación responsiva lograron aceptar mejor los nuevos alimentos.

\section{DISCUSIÓN}

La investigación confirma lo referido por los autores Birch (1998) y Vazir et al. (2013) cuando indican que los niños prefieren alimentos que son ofrecidos en un ambiente positivo. Las acciones realizadas por las cuidadoras modelaron el ambiente de los niños durante la alimentación, es así como las acciones positivas estuvieron asociadas significativamente con la aceptación de los niños a los nuevos alimentos y las acciones de alimentación no responsiva fueron asociadas al rechazo, como lo señala Schroeder et al. (2002). Por ello, el grupo de cuidadoras que aplicaron las técnicas de alimentación responsiva lograron una mejor aceptación de los nuevos alimentos comparadas con las que no las aplicaron, ampliando la variedad de alimentos en la dieta de los niños y acostumbrándolos a que el momento de la alimentación sea agradable y lo disfruten.

Según Mahan y Escott-Stump (2009) los niños en esta etapa están prestos a continuar descubriendo el mundo, atribuyéndole mayor atención a las situaciones poco cotidianas, por ende, disminuyendo su atención por la alimentación. Por esta razón las acciones que resultaron ser las más efectivas en cuanto a la dimensión "anima al niño", estuvieron relacionadas a temas distintos a la alimentación, pues llamaba su atención, creando un ambiente lúdico y poco monótono, necesario para la edad. Además, el niño percibe las sensaciones que presentan las cuidadoras cuando esta se siente cansada o aburrida, la estrategia aplicada en esta situación fue cambiar de cuidadora. Esto creaba un nuevo ambiente positivo y mejoraba la aceptación del alimento.

Las acciones que resultaron efectivas en la segunda dimensión "Hablar con el niño" estuvieron relacionadas al acercamiento y contacto de la cuidadora con el niño. Estas acciones también ayudaron a crear un ambiente positivo para el niño y que este pueda, en el futuro, relacionar su alimentación con un momento grato. Además, al estar con niños sentados en 
una sola mesa se promueve la curiosidad de probar nuevos alimentos, el conversar entre sí e intentar convencer a los compañeros favoreció el consumo de los nuevos alimentos, situación que sucedió durante la investigación en más de una oportunidad, confirmando lo señalado por Mahan y Escott-Stump (2009) y por la Comisión Intersectorial Para la Atención Integral de Primera Infancia (2012), que la comunicación verbal durante la alimentación aumenta la aceptación de los alimentos y estimula el lenguaje de los niños, contribuyendo la mejoría de los hábitos alimentarios.

Sentarse en un lugar destinado a consumir los alimentos y que el niño tenga los alimentos al alcance de su mano contribuye a establecer hábitos alimentarios adecuados, es así que la dimensión "Darle oportunidades al niño para que coma por sí mismo, aunque sea un poco", fue favorable ya que los niños contaron con el entorno físico adecuado pues tenían la posibilidad de comer cómodamente por sí mismos, cumpliendo con esta dimensión, si es que la cuidadora se lo permitía y/o estimulaba; como lo refieren Aboud y Akhter (2011), OPS (2013) y Mahan y Creed (2009). Además, el ofrecimiento de las frutas se realizaba al mismo tiempo para todos y estaban al alcance de sus manos, por lo que tenían mayor oportunidad de comer en forma independiente.

Lacuarta dimensión "ausencia derespuestas inapropiadas a un niño", se encontró que la mayor parte de las cuidadoras que realizaron estas acciones negativas no aplicaron las técnicas de alimentación responsiva; sin embargo, algunas sí lograron que el niño acepte los alimentos, ejecutando una alimentación estricta y poco asertiva. Se observó que, a más respuestas inapropiadas, se reducían los niños que aceptaban los nuevos alimentos y las cuidadoras que aplicaban adecuadamente la alimentación responsiva, excepto en el caso de las acciones correspondientes a los estilos restrictivos, donde los niños aceptaban los alimentos, pero en un ambiente negativo y forzado, sin que las cuidadoras reconozcan el hambre y saciedad de los niños.

Según Aboud, Shafique y Akhter (2009), Aboud y Black (2011) y Bentley, Creed y Wasser (2011), el estilo y modo de la alimentación, como la alimentación responsiva, en los primeros años de edad del niño, cumplen un rol de suma importancia pues estos logran influenciar en la aceptación de los alimentos y la ingesta dietaria, abriendo oportunidades de consumir alimentos variados y a largo plazo mejorando el pronóstico del diagnóstico nutricional. Es así que los niños expuestos a nuevos alimentos lograron aceptarlos mejor con ayuda de la aplicación de las técnicas de la alimentación responsiva por sus cuidadoras, superando a los que no fueron asistidos con las técnicas, pero sí aceptaron los nuevos alimentos. Por lo que la aplicación de las técnicas de alimentación responsiva contribuye a la mejora de hábitos alimentarios a través de la aceptación de nuevos alimentos en la dieta.

\section{CONCLUSIONES}

- La aplicación de las técnicas de alimentación responsiva es un factor que contribuye a la mejora de hábitos alimentarios en niños de 2 a 3 años de edad.

- Las acciones más efectivas en la dimensión "anima al niño" estuvieron 
relacionadas a temas distintos a la alimentación.

- Las acciones que resultaron efectivas en la dimensión "Hablar con el niño" estuvieron relacionadas al acercamiento y contacto de la cuidadora con el niño.

- La dimensión "Darle oportunidades al niño para que coma por sí mismo, aunque sea un poco" resultó efectiva ya que las mesas, sillas y se encontraban al alcance de los niños.

- En la dimensión "ausencia de respuestas inapropiadas a un niño", se encontró que la mayor parte de las cuidadoras que realizaron estas acciones negativas no aplicaron las técnicas de alimentación responsiva.

- Los niños alimentados con ayuda de la aplicación de las técnicas de la alimentación responsiva por sus cuidadoras presentaron mejor aceptación de nuevos alimentos, superando a los que no fueron asistidos con las técnicas. 


\section{REFERENCIAS}

Aboud, F., \& Akhter, S. A. (2011). Cluster-randomized evaluation of a responsive stimulation and feeding intervention in Bangladesh. Pediatrics, 127(5), e1192-e1197.

Aboud, F., \& Black, M., (2011). Responsive feeding is embedded in a theoretical framework of responsive parenting. $J$ Nut. 141(3), 490-494.

Aboud, F., Shafique, S., \& Akhter, S. (2009). A responsive feeding intervention increases children's self-feeding and maternal responsiveness but not weight gain. JNutr., 139(9), $1738-1743$.

Bentley. M., Creed, H., \& Wasser, H. (2011). responsive feeding and child under nutrition in low- and middle-income countries. J JNutr., 141(3), 502-507.

Birch, LL. (1998). Development of food acceptance patterns in the first years of life. Proc Nutr Soc., 57(4), 617-24.

Black, M., y Creed, H. (2012). ¿Cómo alimentar a los niños? La práctica de conductas alimentarias saludables desde la infancia. Rev. Perú Med Exp Salud Pública, 29(3), 373-378.

Comisión Intersectorial para la Atención Integral de Primera Infancia. (2012). Lineamiento técnico de alimentación y nutrición para la primera infancia. Bogotá. Recuperado de http://www.deceroasiempre.gov.co/QuienesSomos/Documents/4.De-Alimentacion-ynutricion-para-la-Primera-Infancia.pdf

Engle, P., Bentley, M., \& Pelto, G. (2000). The role of care in nutrition programmes: current research and a research agenda. Proceedings of the Royal Society, 59, 25-35.

Instituto Nacional de Estadística e Informática. (2010). Indicadores de resultados identificados en los programas estratégicos (2000- 1er. Semestre 2010). Lima: INEI, Dirección Técnica de Demografía e Indicadores Sociales. Recuperado de https://www. inei.gob.pe/media/MenuRecursivo/publicaciones_digitales/Est/Lib0859/Libro.pdf

Instituto Nacional de Estadística e Informática. (2013). Encuesta demográfica y de salud familiar 2012. Lima: INEI. Recuperado de http://proyectos.inei.gob.pe/endes/2012/ Libro.pdf

Instituto Nacional de Salud. (2015). Estado nutricional en niños y gestantes de los establecimientos de salud del Ministerio de Salud. Lima: INS, Dirección Ejecutiva de Vigilancia Alimentaria y Nutricional. Recuperado de http://www.ins.gob.pe/ repositorioaps/0/5/jer/res_2014/INFORME\%20GERENCIAL\%20SIEN\%20 ANUAL\%202014.pdf

Macías, M., Gordillo, S. y Camacho, R. E. (2012). Hábitos alimentarios de niños en edad escolar y el papel de la educación para la salud. Rev. chil. nutr., 39(3), 40-43.

Mahan, K. y Escott-Stump, S. (2009). Krause Dietoterapia (12ª ed.). España: Elsevier.

Organización Panamericana de la Salud. (2009). Alimentación y nutrición del niño pequeño: Memoria de la reunión subregional de los países de Sudamérica, 2-4 diciembre 2008. 
Lima, Perú. Washington, D.C.: OPS. Recuperado de http://www.unicef.org/lac/ Reunion_Sudamericana_de_Alimentacion_y_Nutricion_del_Nino_Pequeno(2).pdf

Organización Panamericana de la Salud (2013). ProPAN: Proceso para la Promoción de la Alimentación del Niño. Guía para el Usuario del Programa de Cómputo Versión 2.0. Washington, DC: OPS. Recuperado de http://apps.who.int/iris/ bitstream/10665/173296/1/ProPAN\%20guia\%20usuario\%20program\%20computo. pdf

Pelto, G., Levitt, E., \& Thairu, L. (2003). Improving feeding practices: current patterns, common constraints, and the design of interventions. Food and Nutrition Bulletin, 24(1), 45-82.

Schroeder, DG., Ha, PB., Bentley, ME., Pachón, H., Sripaipan, T., Caulfield, LE., \& Marsh, DR. (2002). Caregiver styles of feeding and child acceptance of food in rural Viet Nam. Food Nutr Bull., 23(4 Suppl), 95-100.

Vazir, S., Engle, P., Balakrishna, N., Griffiths, PL., Johnson, SL., Creed-Kanashiro, H., ... Bentley, ME. (2013). Cluster-randomized trial on complementary and responsive feeding education to caregivers found improved dietary intake, growth and development among rural Indian toddlers. Matern Child Nutr., 9(1), 99-117.

Fecha de recepción: 15-10-2019

Fecha de aceptación: 28-11-2019 